\title{
Factores predictivos de la microalbuminuria en pacientes pediátricos con diabetes mellitus tipo 1 (DMt1)
}

\author{
Microalbuminuria predictive factors in pediatric patients with type 1 diabetes mellitus
}

(type 1 DM)

\author{
Paola Marianella Pinto Ibárcena ${ }^{1,2}$, Carlos Del Águila Villar ${ }^{1,2}$, María Isabel Rojas \\ Gabulli $^{1,2}$, Juan Falen Boggio ${ }^{1,2}$, Rómulo Lu de Lama ${ }^{1}$, Oswaldo Núñez Almache ${ }^{1,2}$, \\ Eliana Chávez Tejada ${ }^{1}$, Óscar Espinoza Robles ${ }^{1,2}$ \\ Médico Asistente, Servicio de Endocrinología, Instituto Nacional de Salud del Niño, Lima, Perú. \\ ${ }^{2}$ Facultad de Medicina Hipólito Unanue, Universidad Nacional Federico Villarreal, Lima, Perú
}

\begin{abstract}
Resumen
La nefropatía constituye la complicación más grave de la diabetes mellitus tipo 1 (DMt1) siendo una de las manifestaciones iniciales la microalbuminuria. Objetivos: Determinar el comportamiento de los factores epidemiológicos, clínicos y bioquímicos en el desarrollo de microalbuminuria en pacientes con DMt1. Diseño: Estudio de casos y controles. Lugar: Servicio de Endocrinología del Instituto Nacional de Salud del Niño, Lima, Perú. Participantes: Pacientes diabéticos tipo 1 menores de 18 años. Intervenciones: Se estudió 64 pacientes portadores de DMt1, 22 con microalbuminuria y 42 sin esta manifestación, que constituyeron los casos y controles respectivamente. Se consignó como factores epidemiológicos la edad al diagnóstico, tiempo de evolución, género, antecedentes familiares de diabetes, nefropatía, dislipidemia, hipertensión arterial; como factores clínicos, el estado nutricional, desarrollo puberal (Tanner), presión arterial; y como factores bioquímicos, la hemoglobina glicosilada (HbA1c), microalbuminuria y perfil lipídico. Tanto los casos como los controles fueron seguidos durante un año. El análisis estadístico se hizo mediante pruebas de chi cuadrado, odds ratio y regresión logística, para establecer predominancia de factores. Principales medidas de resultados: Estadio puberal, perfil lipídico y HbA1c. Resultados: Los factores de riesgo hallados fueron la presión arterial diastólica elevada $(p=0,037)$, la pubertad $(p=0,008)$, $\mathrm{HbA} 1 \mathrm{c}$ alta $(p<0,0001)$, hipertrigliceridemia $(p=0,007)$ y la hipercolesterolemia $(p<0,0001)$. Conclusiones: La HbA1c elevada, la hipercolesterolemia y la pubertad fueron los factores de riesgo de mayor preponderancia para el desarrollo de microalbuminuria. A fin de prevenir el desarrollo de microalbuminuria, se sugiere buen control metabólico y cuidadoso manejo de la dislipidemia, en especial en pacientes púberes.
\end{abstract}

Palabras clave: Diabetes mellitus insulinodependiente, hemoglobina A glucosilada.

\begin{abstract}
Nephropathy constitutes the most serious type 1 diabetes mellitus (type 1 DM) complication and microalbuminuria is the initial manifestation. Objectives: To determine the role of epidemiological, clinical and biochemical factors in the development of microalbuminuria in type 1 DM patients. Design: Case and control study. Setting: Endocrinology Unit, National Institute of Child Health, Lima, Peru. Patients: Subjects less than 18 year-old with type 1 DM. Interventions: Sixty-four type 1 DM patients were studied, 22 patients with microalbuminuria (cases) and 42 patients without it (controls). Epidemiological factors studied were age at diagnosis, time from onset, gender, family history of diabetes, nephropathy, dyslipidemia, and/or hypertension; clinical factors studied were nutritional status, pubertal stage (Tanner method) and blood pressure; biochemical factors were glycated hemoglobin (HbA1c), mic roalbuminuria and lipid profile. Both cases and controls were followed for one year. Statistical analysis used chi square, odds ratio and multiple logistic regression calculations to determine main risk factors. Main outcome measures: Pubertal stage, lipid profile and HbA1c. Results: Risk factors determined were high diastolic blood pressure $(p=0.037)$, puberty $(p=0.008)$, high $\mathrm{HbA} 1 \mathrm{C}(p<0.0001)$, hypertriglyceridemia $(p=0.007)$, and hypercholesterolemia $(p<0.0001)$. Conclusions: Elevated $\mathrm{HbA} 1 \mathrm{c}$, hypercholesterolemia and puberty were the more important risk factors for development of microalbuminuria. Main measures to prevent development of microalbuminuria were good metabolic control and good management of dyslipidemia, especially in pubertal patients.
\end{abstract}

Key words: Diabetes mellitus/insulindependent, hemoglobin A/glycosylated.

An Fac med. 2012;73(4):293-7

\section{INTRODUCCIÓN}

La diabetes mellitus constituye un problema de salud pública debido a sus efectos sobre la morbimortalidad de la población afectada y las secuelas que ella produce, afectando significativamente la expectativa de vida ${ }^{(1)}$.
Se acepta que entre las complicaciones mayores que suelen afectar a los pacientes con diabetes mellitus se tiene a la nefropatía diabética, en especial en aquellos que presentan diabetes no insulino dependiente o diabetes tipo 2 (DMt2). Los pacientes con DMt1 suelen presentar este tipo de complica- ciones en el proceso evolutivo de la enfermedad, dos a cinco años después de realizado el diagnóstico. De otro lado, el tiempo transcurrido desde el diagnóstico hasta la evidencia del daño renal y proteinuria suele ser 14 a 17 años; y la progresión hasta la insuficiencia renal puede darse entre los 2 y 7 años ${ }^{(1-3)}$. 
El control periódico de la función renal debe incluir la búsqueda de microalbuminuria, así como de factores de riesgo, a fin de retardar la aparición de este tipo de complicación.

Son pocos los trabajos de investigación en nuestro medio que hayan abordado el tema de los factores predictivos de nefropatía diabética en pacientes con DMt; sin embargo, la elevación de la presión arterial sistémica o intrarrenal ${ }^{(4-7)}$, la historia familiar de nefropatía diabética, los años de evolución de la enfermedad ${ }^{(8,9)}$, el mal control glicémico ${ }^{(5,8,10,11)}$, la dislipidemia y el desarrollo puberal $(7,10,12,13)$ han sido asociados con el desarrollo de esta complicación en los pacientes insulinodependientes ${ }^{(1,4,14)}$, los cuales tienen acción relevante en la génesis de esta complicación. A lo cual hay que agregar que existen otros factores no modificables (genéticos y étnicos) que posiblemente contribuyan a incrementar la susceptibilidad para desarrollar proteinuria, y que a pesar de tener un buen control glicémico se desarrolle nefropatía diabética con los años de evolución ${ }^{(1,2,16,17)}$.

El propósito del presente estudio es determinar cuáles son y cómo se comportan los factores epidemiológicos, clínicos y bioquímicos asociados al desarrollo de microalbuminuria en los pacientes con DMt1 que se atienden en el Programa de Atención Integral del Niño Diabético (PAINDI) del Servicio de Endocrinología del Instituto $\mathrm{Na}$ cional de Salud del Niño (INSN); así como, determinar los factores predictivos de microalbuminuria y establecer el o los factores preponderantes para el desarrollo de microalbuminuria en dichos pacientes.

\section{MÉTODOS}

Se ha realizado un estudio de casos y controles, de pacientes menores de 18 años con DMt1, atendidos en la consulta del PAINDI, del Servicio de Endocrinología del INSN.

Para calcular el tamaño de la muestra se usó los parámetros de porcentaje de hemoglobina glicosilada (HbA1c) alta en pacientes diabéticos portadores de microalbuminuria de $80 \%$, y $30 \%$ en los pacientes diabéticos sin microalbuminuria, un poder de prueba de $80 \%$, nivel de confianza de $95 \%$ y relación de 1:2. Se incluyó como casos pacientes menores de 18 años, diagnosticados de DMt1 y portadores de microalbuminuria, con tiempo mínimo de enfermedad de un año de evolución y atendidos de manera regular (dos controles clínicos anuales como mínimo en un intervalo no menor a dos meses y un control bioquímico anual); mientras tanto, los controles no eran portadores de microalbuminuria y tuvieron las mismas características que los casos. Se excluyó a los pacientes diabéticos que presentaban nefropatía no diabética, hipertensión arterial esencial o alguna situación o enfermedad previa que pudiera causar microalbuminuria.

Se definió microalbuminuria como la tasa de excreción de proteína en orina entre 30 y $300 \mathrm{mg} / 24$ horas o 20 a $200 \mathrm{ucg} / \mathrm{min}$, en una muestra de orina de 24 horas; o una concentración de albúmina entre 30 y $300 \mathrm{mg} / \mathrm{mL}$ en una muestra simple de orina ${ }^{(18)}$.

Se revisó las historias clínicas y los datos fueron colectados en una ficha confeccionada para el presente estudio. Se consignó los factores epidemiológicos género, edad al diagnóstico, edad en la última consulta, años de evolución, edad de aparición de la microalbuminuria -para los casos-, antecedentes familiares de diabetes, hipertensión arterial, nefropatía y/o dislipidemia; entre los factores clínicos, peso, talla, índice de masa corporal (IMC) en los mayores de dos años ${ }^{(19)}$, estadio puberal al momento del estudio, presión arterial sistólica y diastólica, según el Task Force de la Academia Americana de Pediatría ${ }^{(20)}$; y como factores bioquímicos, HbA1c (según las normas aprobadas por ISPAD $\left.{ }^{(4)}\right)$, microalbuminuria y perfil lipídico (colesterol total y fraccionado y triglicéridos), según The National Cholesterol Education Program (21).

Se realizó un análisis tomando como variable dependiente a la microalbuminuria y como variables independientes a los factores epidemiológicos, clínicos y bioquímicos, representados en número, porcentaje y promedio. Se determinó la asociación mediante la prueba de chi cuadrado $(p<0,05)$ y se cuantificó mediante el cálculo de los odds ratio. Para establecer la preponderancia de los factores se realizó análisis multivariado a través de regresión logística.

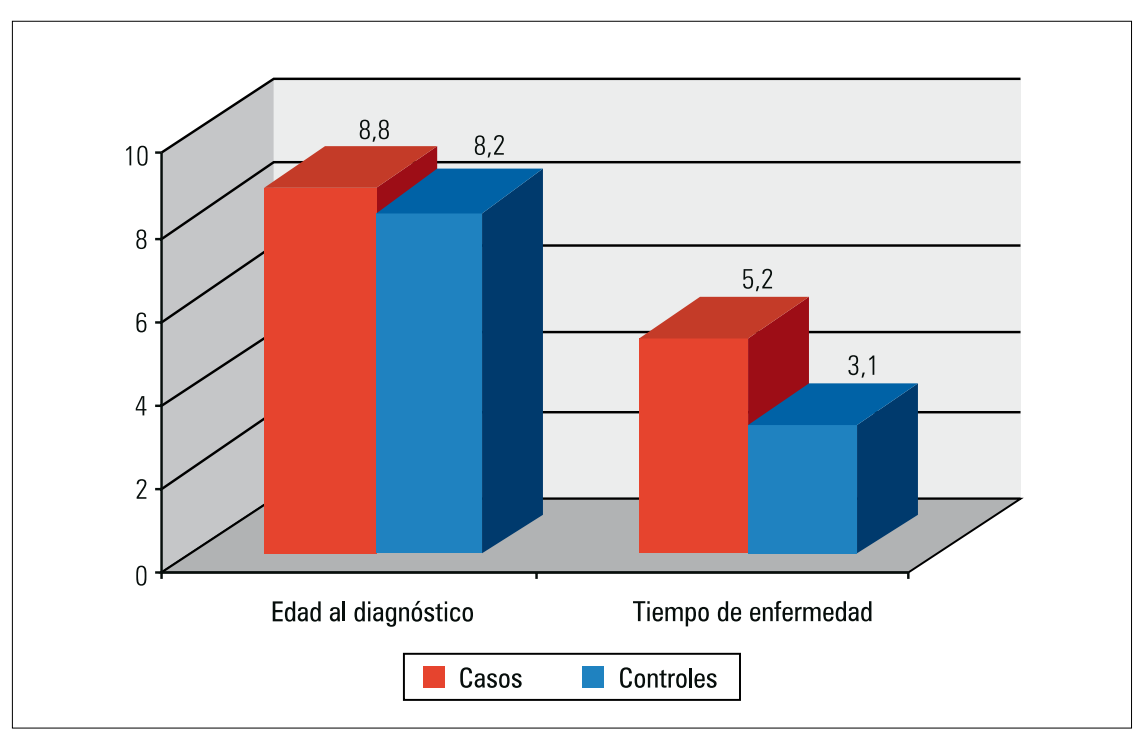

Figura 1. Edad al diagnóstico y tiempo de enfermedad en pacientes diabéticos tipo 1 con y sin microalbuminuria. 
Tabla 1. Antecedentes familiares asociados al desarrollo de microalbuminuria.

\begin{tabular}{|c|c|c|c|c|}
\hline \multicolumn{2}{|c|}{ Antecedentes familiares } & $\begin{array}{c}\text { Casos } \\
(\mathrm{n}=22) \\
n(\%)\end{array}$ & $\begin{array}{c}\text { Controles } \\
(\mathrm{n}=42) \\
\mathrm{n}(\%)\end{array}$ & $p$ \\
\hline Diabetes tipo 2 & $\begin{array}{l}\text { Sí } \\
\text { No }\end{array}$ & $\begin{array}{c}13(59,1) \\
9(40,9)\end{array}$ & $\begin{array}{l}22(52,3) \\
20(47,7)\end{array}$ & $0,804(* *)$ \\
\hline Hipertensión arterial & $\begin{array}{l}\text { Sí } \\
\text { No }\end{array}$ & $\begin{array}{c}3(13,6) \\
19(86,4)\end{array}$ & $\begin{array}{l}13(30,9) \\
29(69,1)\end{array}$ & $0,224(* *)$ \\
\hline Dislipidemia & $\begin{array}{l}\text { Sí } \\
\text { No }\end{array}$ & $\begin{array}{c}7(31,8) \\
15(68,2)\end{array}$ & $\begin{array}{l}13(30,9) \\
29(69,1)\end{array}$ & $1,000(* *)$ \\
\hline Nefropatía & $\begin{array}{l}\text { Sí } \\
\text { No }\end{array}$ & $\begin{array}{c}2(9,1) \\
20(90,9)\end{array}$ & $\begin{array}{c}1(2,4) \\
41(97,6)\end{array}$ & $0,270\left(^{*}\right)$ \\
\hline
\end{tabular}

(*) Prueba exacta de Fisher. (**) Prueba con corrección de Yates.

Tabla 2. Factores clínicos asociados al desarrollo de microalbuminuria.

\begin{tabular}{|c|c|c|c|c|}
\hline \multicolumn{2}{|c|}{ Factores } & $\begin{array}{c}\text { Casos } \\
(\mathrm{n}=22) \\
\mathrm{n}(\%)\end{array}$ & $\begin{array}{c}\text { Controles } \\
(\mathrm{n}=42) \\
\mathrm{n}(\%)\end{array}$ & $p$ \\
\hline Estado nutricional & $\begin{array}{c}\text { Adelgazado } \\
\text { Eutrófico } \\
\text { Sobrepeso/ Obesidad }\end{array}$ & $\begin{array}{c}6(27,3) \\
16(72,7) \\
0(0)\end{array}$ & $\begin{array}{c}5(11,9) \\
35(83,3) \\
2(4,8)\end{array}$ & $0,198\left(^{* *}\right)$ \\
\hline $\begin{array}{l}\text { Presión arterial } \\
\text { sistólica }\end{array}$ & $\begin{array}{l}\text { Normal } \\
\text { Elevada }\end{array}$ & $\begin{array}{c}21(95,5) \\
1(4,5)\end{array}$ & $\begin{array}{c}42(100,0) \\
0(0)\end{array}$ & $0,344(*)$ \\
\hline $\begin{array}{l}\text { Presión arterial } \\
\text { diastólica }\end{array}$ & $\begin{array}{l}\text { Normal } \\
\text { Elevada }\end{array}$ & $\begin{array}{c}19(86,4) \\
3(13,6)\end{array}$ & $\begin{array}{c}42(100,0) \\
0(0)\end{array}$ & $0,037\left(^{* *}\right)$ \\
\hline Estadio puberal & $\begin{array}{l}\text { Pre púber } \\
\text { Púber }\end{array}$ & $\begin{array}{c}2(9,1) \\
20(90,9)\end{array}$ & $\begin{array}{l}19(45,2) \\
23(54,8)\end{array}$ & $0,008\left({ }^{* *}\right)$ \\
\hline
\end{tabular}

${ }^{(*)}$ Prueba exacta de Fisher. $\left.{ }^{\star *}\right)$ Prueba con corrección de Yates.

Tabla 3. Factores bioquímicos asociados al desarrollo de microalbuminuria.

\begin{tabular}{|c|c|c|c|c|c|}
\hline \multicolumn{2}{|c|}{ Factores } & $\begin{array}{c}\text { Casos } \\
(\mathrm{n}=22) \\
\mathrm{n}(\%)\end{array}$ & $\begin{array}{c}\text { Controles } \\
\begin{array}{c}(\mathrm{n}=42) \\
\mathrm{n}(\%)\end{array}\end{array}$ & $p$ & $\mathrm{OR}$ \\
\hline $\mathrm{HbA1c}$ & $\begin{array}{l}\text { Normal } \\
\text { Elevada }\end{array}$ & $\begin{array}{c}1(4,6) \\
21(95,4)\end{array}$ & $\begin{array}{l}22(52,4) \\
20(47,6)\end{array}$ & $0,000\left({ }^{* *}\right)$ & 23,1 \\
\hline Triglicéridos & $\begin{array}{l}\text { Normal } \\
\text { Elevado }\end{array}$ & $\begin{array}{c}4(18,2) \\
18(81,8)\end{array}$ & $\begin{array}{l}24(57,1) \\
18(42,9)\end{array}$ & $0,007\left(^{* *}\right)$ & 6,0 \\
\hline Colesterol total & $\begin{array}{l}\text { Normal } \\
\text { Elevado }\end{array}$ & $\begin{array}{c}2(9,1) \\
20(90,9)\end{array}$ & $\begin{array}{l}25(59,5) \\
17(40,5)\end{array}$ & $0,000\left({ }^{* *}\right)$ & 14,706 \\
\hline Colesterol LDL & $\begin{array}{l}\text { Normal } \\
\text { Elevado }\end{array}$ & $\begin{array}{c}3(13,6) \\
19(86,4)\end{array}$ & $\begin{array}{l}23(54,8) \\
19(45,2)\end{array}$ & $0,004\left({ }^{* *}\right)$ & 7,667 \\
\hline Colesterol HDL & $\begin{array}{c}\text { Normal } \\
\text { Disminuido }\end{array}$ & $\begin{array}{c}1(4,6) \\
21(95,4)\end{array}$ & $\begin{array}{c}6(14,3) \\
36(85,7)\end{array}$ & $0,445\left(^{* *}\right)$ & 3,5 \\
\hline
\end{tabular}

HbA1C = hemoglobina glicosilada. $\left.{ }^{\star \star}\right)$ Prueba con corrección de Yates.

Tabla 4. Factores predictivos para el desarrollo de microalbuminuria en pacientes diabéticos tipo 1.

\begin{tabular}{|c|c|c|c|c|}
\hline \multirow{2}{*}{ Factores } & \multirow{2}{*}{$p$} & \multicolumn{2}{c|}{ OR } & \multicolumn{2}{c|}{ LC (OR) 95\% } \\
\cline { 4 - 5 } HbA1C & 0,011 & 18,071 & 1,945 & 167,934 \\
\hline Colesterol total & 0,014 & 9,030 & 1,564 & 52,135 \\
\hline Estado puberal & 0,021 & 8,231 & 1,379 & 49,147 \\
\hline
\end{tabular}

Regresión logística multivariada. $\mathrm{HDA} 1 \mathrm{C}=$ hemoglobina glicosilada. $\mathrm{LC}=$ límite de confianza.

\section{RESULTADOS}

Se evaluó 64 pacientes menores de 18 años con DMt1, 22 con microalbuminuria $(34,4 \%)$ y 42 sin microalbuminuria $(65,6 \%)$. El $50 \%$ fue de sexo masculino y el otro $50 \%$ de sexo femenino, tanto para casos como para controles. La edad promedio al momento del estudio fue 14,1 años (DE 2,6) para los casos y 11,4 años (DE 3,2) para los controles.

En la figura 1 se muestra la edad al diagnóstico y el tiempo de enfermedad para los casos y controles, observándose que para los casos, la edad al momento del diagnóstico de DMt1 fue en promedio 8,8 años (rango: 3,0 a 14,5; DE 3,0) y para los controles 8,2 años (rango: 0,17 a 13,75; DE 3,5). Respecto al tiempo de enfermedad transcurrido, este tuvo un promedio de 5,2 años (DE 2,6) para los casos y 3,1 años (DE 1,9) para los controles $(p<0,0001)$.

La edad al momento de diagnosticarse microalbuminuria fue 11,8 años (rango: 7,25 a 17,17; DE 3,1), mientras que el tiempo transcurrido desde el diagnóstico de DMt1 hasta el hallazgo de microalbuminuria fue 3,02 años (DE $3,1)$.

En la tabla 1 se muestra los antecedentes familiares, observándose que no existió asociación significativa.

En la tabla 2 se muestra los factores clínicos asociados, observándose significancia estadística en la presión arterial diastólica elevada (13,6\% de los casos y $0 \%$ de los controles) y en la condición de púber $(90,9 \%$ de los casos y $54,8 \%$ de los controles).

En la tabla 3 se observa los factores bioquímicos asociados; $95,4 \%$ de los casos registró valores de $\mathrm{HbA1c}$ elevados frente a $47,6 \%$ de los controles, mientras que respecto al perfil lipídico se tuvo valores elevados de colesterol total LDL-C y triglicéridos y valores disminuidos para el HDL-C en los portadores de microalbuminuria.

En la tabla 4, se nota que la calidad del control glicémico medido por la 
media global de la HbA1c (OR: 18,071 LC-95\%: 1,94 a 167,93), la media global del valor del colesterol total (OR: 9,030 LC: $1,56 \%$ a $52,13 \%$ ) y la pubertad (OR: 8,23 LC: 1,37 a 49,14) estuvieron relacionadas significativamente con la aparición de microalbuminuria.

\section{DISCUSIÓN}

La nefropatía diabética ha aumentado de manera alarmante en los últimos años, considerándose un problema de magnitud mundial ${ }^{(10,12,22)}$. La microalbuminuria, como expresión de daño renal, se ha correlacionado también con disfunción endotelial, retinopatía y enfermedad cardiovascular ${ }^{(1,23)}$. De 10 a 30\% de los DMt1 desarrollarán microalbuminuria en los primeros cinco años de la enfermedad y, sin intervención terapéutica, $80 \%$ de quienes desarrollan microalbuminuria aumentará su excreción, llegando en 10 a 15 años a la fase de nefropatía clínica y, de ellos, en otros 10 años el 50\% llegará a insuficiencia renal terminal ${ }^{(13,23)}$.

En nuestro estudio no encontramos diferencia estadísticamente significativa respecto a la edad en que se diagnosticó la DM1, a diferencia de Bogdanovic y Bustillo Solano ${ }^{(7,16)}$ que señalan que el riesgo de desarrollar nefropatía diabética es mayor cuando la enfermedad aparece antes de los 11 años. Schultz y colaboradores ${ }^{(25)}$ encuentran que $45 \%$ de los pacientes con debut antes de los 5 años desarrolla microalbuminuria antes de la pubertad. Raile y colaboradores ${ }^{(14)}$ señalan el inicio en edad infantil como factor protector.

El tiempo de enfermedad más prolongado mostró en nuestro estudio ser un factor fuertemente predictivo para el desarrollo de microalbuminuria, concordando con lo dicho por Cooper ${ }^{(8)}$, Mongensen ${ }^{(9)}$, Bustillo Solano ${ }^{(7)}$, Pérez G. y colaboradores ${ }^{(15)}$.

Por otro lado, a diferencia de lo descrito por Raile y colaboradores ${ }^{(14)}$, quienes encuentran que el desarrollo de microalbuminuria se asocia al sexo masculino, en el presente estudio no hallamos asociación alguna. De manera similar, los antecedentes familiares de diabetes, hipertensión, dislipidemia y nefropatía no mostraron ser factores predictivos para el desarrollo de microalbuminuria.

En relación al estado nutricional como factor clínico asociado, en ambos grupos de estudio, la mayor proporción de pacientes fue eutrófica, lo que revela que un aparente buen estado nutricional, por antropometría, no garantiza protección frente al desarrollo de microalbuminuria.

Viberti y colaboradores (22) mostraron que el incremento de la excreción de albúmina urinaria precede al aumento de la presión arterial. Nuestra investigación demostró que 13,6\% de los casos presentaba elevación de la presión arterial diastólica; no se pudo precisar si la elevación de la presión precedió a la microalbuminuria; el porcentaje es importante si consideramos que el tiempo promedio de enfermedad era de 3 años.

Se encontró que la pubertad fue un factor de riesgo predictivo ocho veces mayor para desarrollar microalbuminuria, coincidiendo con diversas publicaciones, atribuyéndose participación fisiopatológica en el desarrollo de las complicaciones microvasculares a la hormona de crecimiento y los esteroides sexuales ${ }^{(1,7,10,12-14)}$.

El nivel de HbA1c está estrechamente relacionado con el inicio y progresión de las complicaciones microvasculares ${ }^{(1,7,14,28)}$. En el presente estudio, la estimación de la probabilidad de desarrollar microalbuminuria fue veintitrés veces mayor cuando la $\mathrm{HbAlc}$ estuvo elevada, similar a lo señalado por Nordwall (29), James ${ }^{(28)}$, Parving ${ }^{(3)}$ y otros ${ }^{(1,7,14)}$, quienes han precisado que concentraciones de $\mathrm{HbA} 1 \mathrm{c}$ superiores a $8,5 \%{ }^{(16)}, 9 \%^{(18)}$ o $10 \%{ }^{(4)}$ confieren susceptibilidad para incrementar la excreción de albumina urinaria.

Si bien los resultados del ensayo de control de la diabetes y sus complica- ciones (DCCT) ${ }^{(18)}$ indican que una hemoglobina dentro del 95 percentil de sujetos no diabéticos pudiera ser necesaria para evitar el desarrollo de cualquier complicación microvascular y que el estudio prospectivo de Reino Unido (UKPDS) demuestra claramente que sin importar cómo se alcance el control de la glicemia existe una clara reducción del riesgo de complicaciones de la diabetes mellitus incluyendo la nefropatía, se pone en evidencia que debe existir una situación de descontrol muy prolongada y que otros factores no controlados (genéticos, raciales) posiblemente determinen la susceptibilidad de desarrollar microalbuminuria; así, nuestros hallazgos deben llevar a revisar las guías de evaluación y seguimiento de nuestra población con DMt1, a fin de considerar otros aspectos además del control metabólico.

En relación al perfil lipídico, encontramos que la hipertriglceridemia, el colesterol total y LDL-C elevados fueron factores predictivos para la progresión a microalbuminuria. Al respecto, la literatura ha atribuido un posible papel a la concentración elevada de la fracción aterogénica (LDL-C) o a la reducida relación de HDL-C/LDL-C en la patogénesis de la enfermedad microvascular diabética, incluyendo a la nefropatía diabética ${ }^{(3,30)}$. Sin embargo, Ekberg y colaboradores ${ }^{(31)}$ no hallan evidencia clínica ni bioquímica de la existencia de una posible asociación entre hipercolesterolemia como posible factor de riesgo para el desarrollo de nefropatía diabética. Así mismo, Bustillo Solano ${ }^{(7)}$ sugiere que el perfil lipídico no constituye un factor de riesgo relevante para el desarrollo de microalbuminuria, y que más bien las alteraciones lipídicas en el paciente con DMt1 pueden deberse a la complicación renal; aceptando que las alteraciones en el metabolismo de las lipoproteínas podrían convertirse con el tiempo en un perpetuador de la enfermedad renal diabética. A diferencia de lo descrito en este estudio, Pérez A ${ }^{(33)}$ y colaboradores encuentran que el desorden dislipidémico más frecuente en pacientes diabéticos tipo 1 con pobre 
control metabólico es el HDL-C bajo. Nuestro estudio coincide con Torres $\mathrm{M}^{\text {(32) }}$ y colaboradores, en referencia a que pacientes con buen control metabólico tienen perfiles lipídicos similares a sus pares no diabéticos; lo que apoya la hipótesis de que el pobre control glicémico se asocia a perfiles lipídicos aterogénicos.

En conclusión, nuestro estudio encontró como factores predictivos preponderantes para el desarrollo de microalbuminuria a los valores elevados de $\mathrm{HbA1c}$, la hipercolesterolemia y la aparición de pubertad.

En relación a la prevención del daño renal, consideramos que el cuidado de la diabetes debe enfocarse en el buen control metabólico a largo plazo y en la reducción de factores de riesgo adicionales, como la dislipidemia.

\section{REFERENCIAS BIBLIOGRÁFICAS}

1. Pombo M. Tratado de Endocrinologia Pediátrica, 3ra Edición, Mc Graw Hill- Interamericana. 2002:1560-603.

2. Nelson RG, Bennett PH, Beck GJ, Tan M, Knowler WC, Mitch WE, et al. Development and progression of renal disease in Pima Indians with noninsulin-dependent diabetes mellitus. N Engl J Med. 1996;335:1636-42.

3. Parving $\mathrm{H}-\mathrm{H}$. Diabetic nephropathy: prevention and treatment. Kidney Int. 2001;60:2041-55.

4. Rewers M, Pihoker C, Dohaghue K, Hanas R, Swift $P$, Klingensmith GJ. Assessment and monitoring of glycemic control om children and adolescents with diabetes. ISPAD Clinical Practice Consensus Guidelines 2009 Compendium. Chapter 7. Pediatric Diabetes. 2009:10(12):71-81

5. Zubiate M. Epidemiologia de la diabetes mellitus en el Perú. Diagnóstico. 1996;32(1):22-40.

6. Carvajal C, Márquez PE, Licea ME, Arranz C Güell R. Excreción urinaria de albúmina en niños diabéticos insulinodependientes. Rev Cubana Endocrinol. 1996;(7)1.

7. Bustillo S. Variables predictoras en la proteinuria en los pacientes diabéticos insulinodependientes. Rev Cub Endocrinol. 1998;9(2):123-31.
8. Cooper ME. Pathogenesis, prevention, and treatment of diabetic nephropathy. Lancet. 1998;352(9123):213-9.

9. Mogensen CE, Christensen CK. Predicting diabetic nephropathy in insulindependent patients. N Engl J Med. 1984;311(2):89-93.

10. Battilana C, Lu G. Nefropatía diabética. Diagnóstico. 2000;39(2):66-79.

11. Hernández FC, Villatoro G. Nefropatía diabética en pacientes que asisten a la consulta externa de endocrinologia pediátrica del Hospital Escuela. Rev Méd de los Post Grad Med UNAH. 2007;10(1).

12. Argente $O$, Carrascasa L, Garcia B, Rodriguez H. Tratado de Endocrinología Pediátrica y de la Adolescencia. Segunda edición, Barcelona: Doima 2000:1268-71.

13. Gallardo TV, Ugarte P. Pesquisa precoz de nefropatía diabética en niños y adolescentes portadores de diabetes mellitus tipo 1. Arch Pediatr Urug. 2007;78(3):247-52.

14. Raile K, Galler A, Hofer S. Diabetic nephropathy in 27,805 children, adolescents, and adults with type 1 diabetes. Effect of diabetes duration, A1C, hypertension, dyslipidemia, diabetes onset, and sex. Diabetes Care. 2007;30(10):2523-8.

15. Pérez GR, Rodríguez P, Dall'Anesse C, Gómez $F$, Valderrabano F. Preocupante incremento de la diabetes como causa de insuficiencia renal terminal. Evaluación del tratamiento sustitutivo. An Med Interna. 2001;18:175-80.

16. Bogdanovic R. Diabetic nephropathy in children and adolescents. Pediatr Nephrol. 2008;23:50725.

17. Lu R, Dextre A, Falen J, Del Aguila C, Rojas MI, Nuñez O, et al. Características clinicas y sociales de 105 niños diabéticos del Instituto de Salud del Niño. Diagnóstico. 2000;38(5).

18. The Diabetes Control and Complications Trial Research Group. The effect of intensive treatment of diabetes on the development and progression of long-term complications in insulin-dependent diabetes mellitus. N Engl J Med. 1993;329:977-86.

19. Frankel HM. Body mass index graphics for children. Pediatrics. 2004;113(2):425-6.

20. The fourth report on the diagnosis, evaluation, and treatment of high blood pressure in children and adolescents. Pediatrics. 2004;114(2):555-76.

21. National Cholesterol Education Program. Highlights of the report of the Expert Panel on Blood Cholesterol Levels in Children and Adolescents. Pediatrics. 1992;89:495-501.

22. Viberti $G$, Keen $H$. The patterns of proteiunuria in diabetes mellitus. Relevance to pathogenesis and prevention of diabetic nephropathy. Diabetes. 1984;33:686-92.

23. Rakesh A, Barry W, Prevost T. Risk of microalbuminuria and progression to macroalbuminuria in a cohort with childhood onset type 1 diabetes: prospective observational study. BMJ. 2008;336:697-70.
24. Drummond K, Mauer M. International Diabetic Nephropathy Study Group. The early natural history of nephropathy in type 1 diabetes. Early renal structural changes in type 1 diabetes. Diabetes. 2002;51(5):1580-7.

25. Schultz CJ, Dalton R, Dunger D. Risk of nephropathy can be detected before the onset of microalbuminuria during the early years after diagnosis of type 1 diabetes. Diabetes Care. 2000;23(12):1811-5.

26. Mogensen CE. Microalbuminuria, blood pressure and diabetic renal disease: origin and development of ideas. Diabetología. 1999;42:263-85.

27. Caramori ML, Fioretto P, Mauer M. The need for early predictors of diabetic nephropathy risk. Is albumin excretion rate sufficient? Diabetes. 2000;49(9):1399-408.

28. Nordwall M, Arnqvist HJ, Bojestig M. Good glycemic control remains crucial in prevention of late diabetic complications. The Linkoping Diabetes Complications Study. Pediatr Diabetes. 2009;10(3):168-76.

29. James H, Laura JS, Linda SH, Wantman M, Cohen S, Laff M. Progression of microalbuminuria to proteinuria in type 1 diabetes nonlinear relationship with hyperglycemia. Diabetes. 2000;49(1):94-100.

30. Eckel RH, McLean E, Albers JJ, Cheung MC, Berman EL. Plasma lipids and microangiopathy in insulin-dependent diabetes mellitus. Diabetes Care. 1981;4:447-53.

31. Ekberg G, Grefberg N, Larsson O, Vara I. Relation between serum cholesterol and diabetic nephropathy. Lancet. 1990;336(abst):954.

32. Torres M, Lerman I, Bravo LE, Cardoso G, Mendoza F, Zamora J, et al. Metabolic control and the prevalence of dyslipidemia in children and adolescents with insulin-dependent diabetes mellitus. Rev Invest Clin. 1993;45(6):545-52.

33. Pérez A, Wagner AM, Carreras G, Giménez G, Sánchez-Quesada JL, Rigla M, et al. Prevalence and phenotypic distribution of dyslipidemia in type 1 diabetes mellitus: effect of glycemic control. Arch Intern Med. 2000;160(18):2756-62.

Artículo recibido el 1 de mayo de 2012 y aceptado para publicación el 14 de junio de 2012.

Correspondencia:

Dra. Paola Marianella Pinto Ibárcena

Pasaje Quiñones 104 Dpto. 404, Jesús María

Correo electrónico: paolapinto2003@yahoo.es 